OPEN ACCESS

Edited by:

Heikki Helanterä,

University of Oulu, Finland

Reviewed by:

Mark Bulmer

Towson University, United States

Aya Yanagawa,

Kyoto University, Japan

*Correspondence:

Dino P. McMahon

dino.mcmahon@fu-berlin.de

Specialty section:

This article was submitted to

Social Evolution

a section of the journa

Frontiers in Ecology and Evolution

Received: 23 October 2019

Accepted: 05 March 2020

Published: 03 April 2020

Citation:

Esparza-Mora MA, Davis HE,

Meconcelli S, Plarre $R$ and McMahon DP (2020) Inhibition of a Secreted Immune Molecule Interferes

With Termite Social Immunity.

Front. Ecol. Evol. 8:75.

doi: $10.3389 /$ fevo.2020.00075

\section{Inhibition of a Secreted Immune Molecule Interferes With Termite Social Immunity}

\author{
M. Alejandra Esparza-Mora ${ }^{1,2}$, Hannah E. Davis ${ }^{3}$, Stefania Meconcelli $^{2}$, Rudy Plarre ${ }^{2}$ and \\ Dino P. McMahon ${ }^{1,2 *}$
}

${ }^{1}$ Institut für Biologie, Freie Universität Berlin, Berlin, Germany, ${ }^{2}$ Abteilung 4 Material und Umwelt, Bundesanstalt für Materialforschung und -prüfung (BAM), Berlin, Germany, ${ }^{3}$ Department of Biology, Carleton University, Ottawa, ON, Canada

Social immune behaviors are described in a great variety of insect societies and their role in preventing emerging infectious diseases has become a major topic in insect research. The social immune system consists of multiple layers, ranging from the synthesis of external immune molecules to the coordination of individual behaviors into sophisticated collective defensive tasks. But our understanding of how complex group-level behavioral defenses are orchestrated has remained limited. We sought to address this gap in knowledge by investigating the relationship between the external activity of an important immune effector molecule in termites, Gram negative binding protein 2 (GNBP-2) and collective grooming and cannibalism. We reasoned that as an external enzyme capable of degrading entomopathogenic fungi, GNBP-2 can facilitate the spread of pathogenic molecules in the colony, and thus serve to trigger collective defenses in a manner analogous to pathogen-associated molecular signatures (PAMPs) of the individual immune system. To test whether GNBP-2 could play a role in regulating social immune behavior, we experimentally inhibited its fungicidal activity using the glycomimetic molecule, D-d-gluconolactone (GDL) and recorded collective behavioral responses to an infected nestmate. Contrary to expectations, GNBP-2 inhibition did not influence the rate or intensity of grooming of either control or fungus-infected nestmates. By contrast, we found that the probability of being harmed through defensive cannibalistic behaviors was significantly reduced by the inhibition of GNBP-2. Our findings indicate that the regulation of collective immune behaviors may depend in part on the external secretion of an enzyme originating from the individual immune system, but that other cues are also necessary.

Keywords: termite, social immunity, cannibalism, hygienic behavior, GNBP-2, GDL, Metarhizium, entomopathogen

\section{INTRODUCTION}

The evolutionary and ecological success of social insects can in large part be attributed to the evolution of division of labor. However, sociality also poses specific disadvantages, including increased exposure of colonies to infectious diseases (Richard, 1974; Cremer et al., 2018). The apparent disease susceptibilities associated with social live have imposed significant selection 
pressures on social insects to regulate the emergence and spread of disease (Schmid-Hempel, 1995; Cremer et al., 2007; Meunier, 2015). This may help to explain why epizootics that can kill entire social insect colonies are in fact quite rare (Chouvenc and Su, 2012; Schmid-Hempel, 2017). Social insect individuals are able to limit infection using their individual immune systems (Cotter and Kilner, 2010; Meunier, 2015) but they have also evolved a variety of collective disease defenses to mitigate the occurrence and dissemination of infectious diseases (Cremer et al., 2007; Wilson-Rich et al., 2009) including both behavioral and physiological adaptations (Cremer et al., 2018; Bulmer et al., 2019; Liu et al., 2019). Social actions resulting in the control or elimination of infections are examples of "social immunity." Social immunity combines defenses exhibited by the host with defenses that can be generated by surrounding relatives (Van Meyel et al., 2018). Social immunity has been termed a key property of social system evolution (Cremer et al., 2018), although a unique link between social immunity and true sociality has recently been questioned (Van Meyel et al., 2018).

Despite growing interest in the study of social immunity, we remain far from understanding how collective defensive behaviors are regulated. This is partly because social immunity represents a "distributed organ" that is comprised of a diverse array of defensive traits. For example, externally-secreted molecules derived from the individual immune system, such as toxins, acids and peptides often operate in conjunction with collective behavioral responses to protect groups against infection (Hamilton et al., 2011; Otti et al., 2014), with such molecules likely serving a critical role as a primary barrier to infection (Zasloff, 2002). In ants, termites as well as other social insect groups, behavioral defenses can be supplemented with the secretion and spread of antimicrobial substances onto body surfaces, where they function as a potent external disinfectants (Hamilton et al., 2011; López-Riquelme and Fanjul-Moles, 2013; Otti et al., 2014; He et al., 2018; Pull et al., 2018). Termites in particular can deploy a wide repertoire of social immune responses including alarm behaviors, avoidance, prophylactic, or antimicrobial secretions, burial of dead bodies, necrophagy, mutual grooming, and cannibalism (Rosengaus et al., 1998, 1999, 2011; Yanagawa and Shimizu, 2007; Chouvenc et al., 2008; Chouvenc and Su, 2010; He et al., 2018; Bulmer et al., 2019). Antimicrobial secretions in termites are produced by sternal as well as head glands, and can include antimicrobial compounds found in rectal fluids and feces (Rosengaus et al., 2011; Bulmer et al., 2019).

Termites therefore represent an excellent eusocial model for studying the evolution and function of animal immunity and sociality. However, understanding when and why different collective defenses are deployed in response to an infectious disease threat remains a significant challenge to research. We recently showed that termites can employ a range of collective responses depending on the individual's progression along the stepwise-infection process (Davis et al., 2018). But we do not understand the underlying mechanism(s) responsible for regulating the point at which these different responses are deployed. Here, we chose to examine whether immune components synthesized and secreted by individuals could play an important role in regulating group-level behavioral responses to disease. Specifically, we focus on the role of the Gramnegative bacteria binding protein 2 (GNBP-2), which alongside the peptide Termicin, has received particular attention in termite immunity research (Lamberty et al., 2001; Yuki et al., 2008; Bulmer et al., 2009). Termicins are a class of antimicrobial peptides (AMPs) with strong antifungal activity, while GNBP-2 belongs to a class of bifunctional pattern recognition receptors (PRRs) that can recognize lipopolysaccharide (LPS) of Gramnegative bacteria and $\beta$-1,3-glucans of fungal cell walls (Bulmer et al., 2009; Hamilton and Bulmer, 2012). These proteins were first described in Nasutitermes (Bulmer et al., 2009) and later in Reticulitermes (Hamilton et al., 2011; Hamilton and Bulmer, 2012). The $\beta$-1,3-glucanase activity of termite GNBP-2 can protect termites against lethal infection by damaging conidia cell walls and thereby inhibiting germination (Rosengaus et al., 2014). GNBP-2 has been found on the insect cuticle after allogrooming as well as in nest materials, where it is likely to provide protection against generalist pathogenic fungi found in the colony environment (Bulmer et al., 2009; Hamilton et al., 2011; Hamilton and Bulmer, 2012). GNBP-2 mediated release of digested $\beta$-1,3-glucans or other fungal cell components into the nest environment could help to prevent the spread of infection by eliciting an immune response in (and thereby priming the immune defenses of) uninfected nearby termites (Bulmer et al., 2009; Hamilton and Bulmer, 2012). In addition to putative roles in termite external immunity, GNBP-2 is known to occur in the termite alimentary canal where it may act as an internal disinfectant and serve to regulate gut symbiosis during digestion (Yuki et al., 2008).

While inactivation of GNBP-2 results in suppressed immune defenses at the individual level, its involvement in collective behavior is unknown. We hypothesize that by facilitating the degradation and spread of fungal cell wall components, GNBP2 could act as a signal amplifier within the colony, serving to recruit nestmates to the source of infection, and therefore acting as an important molecular cue for collective defensive behavior. We test whether GNBP-2 can act as a molecular mediator of collective defense behavior by experimentally inhibiting the $\beta$ 1,3-glucanase activity with D-d-gluconolactone (GDL) (Bulmer et al., 2009; Hamilton et al., 2011) and recording collective behavioral responses to nestmates infected with the fungal entomopathogen Metarhizium anisopliae. Entomopathogenic fungi such as Metarhizium have been important microorganisms in the study of insect social immunity (Rosengaus et al., 1998; Yanagawa et al., 2008; Konrad et al., 2012, 2018; Chouvenc and Su, 2012). These pathogens infect insects via cuticular penetration, leading to host death and the subsequent production of a large number of infectious spores (Vega et al., 2012; Mora et al., 2017). As facultative pathogens, they are widespread in the environment surrounding insect colonies (Cremer et al., 2018). We selected M. anisopliae for use in our experiments as it is a natural pathogen of termites including Reticulitermes flavipes (Zoberi, 1995; Dong et al., 2007) and has served as an effective model entomopathogen in the study of virulence and termite immune defense (Chouvenc et al., 2009; Chouvenc and Su, 2010; Hamilton and Bulmer, 2012; Davis et al., 2018). 


\section{METHODS}

\section{Insect Hosts}

Three $R$. flavipes colonies were used in experiments: colonies $11+13,10$, and $\mathrm{X}$. Pieces of wood containing dense aggregations of termites belonging to these colonies were collected from the field. Colonies $11+13$ and 10 were collected in Île d'Oléron, France, in 1999 and 1994 respectively and maintained in a dark room at $26^{\circ} \mathrm{C}, 84 \%$ humidity. Colony $\mathrm{X}$ was collected in 2015 in Soulac-sur-Mer, France. It was maintained in a dark room at $28^{\circ} \mathrm{C}, 83 \%$ humidity. Primary reproductives of $R$. flavipes can live to 18 years in the wild and up to 25 years in captivity (Lainé and Wright, 2003). Furthermore, secondary reproductives, which can breed amongst themselves, frequently replace primary reproductives in both native and invasive populations of this species, meaning that high levels of inbreeding are not uncommon in R. flavipes (Vargo and Husseneder, 2009). Colonies were kept in separate sheet metal tanks as described by Becker (1969) and had access to wood as well as sufficient damp soil to burrow. Cardboard baits were used to extract termites from their parent colonies according to Tracy (2003). After collection, we maintained termites derived from the same colony inside plastic boxes containing cellulose pads (Pall Corporation, Port Washington, United States) that had been moistened with tap water. Collected termites were kept at the same temperature as the parent colony until they were used for the experiment.

\section{Preparation of Petri Dish Nests}

The Petri dish nest was built as described elsewhere (Davis et al., 2018) to house experimental mini-colonies of $R$. flavipes. The petri dish experimental nest $(94 \times 16 \mathrm{~mm})$ contained two cellulose pads ( $45.5 \mathrm{~mm}$ diameter, $0.9 \mathrm{~mm}$ thick) (Pall) which were placed on top of the two thin filter paper disc Whatman No. 5 (47 mm diameter, $0.2 \mathrm{~mm}$ thick). A standard microscope slide made of glass $(76 \times 26 \mathrm{~mm})$ was then placed on top of all the filter papers. In every Petri dish, we introduced 49 healthy termites (not including the focal individual): 48 medium-to-large workers (3-5 mm body length) and one soldier. Experimental nests were sealed with parafilm to maintain a high level of humidity within petri dishes, and left in a dark room at $27^{\circ} \mathrm{C}$ and $70 \%$ humidity for 15 days to enable the termites to establish tunnels under the glass. To ensure a clear view into the nest a cotton swab was used to remove debris from the top surface of the glass $24 \mathrm{~h}$ prior to the observation experiment.

\section{Fungal Conidia Preparation}

Preparation of $M$. anisopliae conidia for use in experiments was done following Davis et al. (2018). Briefly, M. anisopliae DSM 1490 was maintained on potato dextrose agar (PDA) at $25^{\circ} \mathrm{C}$ in the dark. The conidia used in experiments were derived from a plate that had undergone a single passage from the frozen stock. Conidia from 15 days old cultures were harvested by scraping off the conidia with a sterile cotton swab moistened with sterile $0.05 \%$ Tween 80 and suspending them in sterile $0.05 \%$ Tween 80 solution. The suspension was vortexed for $30 \mathrm{~s}$, then filtered through a piece of sterile miracloth (Merck KGaA, Darmstadt,
D). Filtering removes hyphae and large clumps of conidia from the suspension. The filtered conidia were centrifuged for $10 \mathrm{~min}$ at 5,000 $\mathrm{g}$ at $4^{\circ} \mathrm{C}$ and the pellet was resuspended and washed three times with sterile $0.05 \%$ Tween 80 , with repeated centrifugation (10 min at 5,000 $\mathrm{g}$ at $4^{\circ} \mathrm{C}$ ) between each washing step. Conidia concentration was estimated in a BLAUBRAND Thoma counting chamber (depth $0.1 \mathrm{~mm}$; BRAND, Wertheim, Germany) and the conidia suspension was adjusted to a final concentration of $1 \times 10^{8}$ conidia/mL and stored at $4^{\circ} \mathrm{C}$ until use. Conidia viability following lab culturing was evaluated by streaking with $10 \mu \mathrm{L}$ of the same $1 \times 10^{8}$ conidia/mL suspension and incubating at $25^{\circ} \mathrm{C}$ in the dark. After $21 \mathrm{~h}$ of inoculation, at least 300 conidia per plate were evaluated for germination. A conidium was considered germinated if the length of the germ tube was at least half the diameter of the conidium. The germination rate was $>95 \%$ for all experiments.

\section{Infection With Conidia or $\mathbf{0 . 0 5 \%}$ Tween $\mathbf{8 0}$}

We marked focal termites with Nile blue dye in order to differentiate them from colony nestmates. Nile blue dyeing was carried out following a rapid method for marking termites as described previously (Davis et al., 2018), adapted from Evans (2000). Termite workers were dipped into $2 \mathrm{~mL}$ microcentrifuge tubes and a sufficient quantity of $0.025 \%$ Nile blue (diluted in distilled water) was added to ensure they were completely covered. Focal termites were gently mixed for $1 \mathrm{~min}$, then tipped out onto a dry Whatman No. 1 filter paper disc $(90 \mathrm{~mm}$ diameter, $0.18 \mathrm{~mm}$ thick). Termites were transferred to small plastic containers, one per colony, each containing cellulose pads moistened with tap water, once they had recovered sufficiently to be able to walk. The plastic containers containing the focal termites were closed with a red tight-fitting lid to prevent desiccation and were left overnight in a dark room at $27^{\circ} \mathrm{C}$ and $70 \%$ humidity. Nile blue-marked termites were immersed in $1 \times 10^{8}$ conidia/mL suspension for $10 \mathrm{~s}$ and then allowed to dry onto a Whatman No. 1 filter paper disc. Infected termites were transferred individually into separate small $(35 \mathrm{~mm})$ Petri dishes, each containing a cellulose pad moistened with $1 \mathrm{~mL}$ tap water. Control termites were handled similarly but using a conidia-free solution sterile $0.05 \%$ Tween 80 . The infected and control termites were incubated for $12 \mathrm{~h}$ at $25^{\circ} \mathrm{C}$ before use in the behavioral experiment. This incubation time point was chosen based on a previous study that explored termite collective behavioral responses to termites at different stages of infection (Davis et al., 2018). At $12 \mathrm{~h}$ post-infection, the authors recorded significantly elevated levels of allogrooming performed by nestmates, followed by a gradual transition to cannibalism, as the infected termites began to show visible signs of sickness. The 12-h incubation time point therefore represents an optimal stage during $M$. anisopliae infection to measure the impact of treatment on two essential nestmate behaviors (i.e., allogrooming and cannibalism).

\section{Inhibition of GNBP-2}

D-d-gluconolactone (GDL) was used to block the activity of termite gram-negative binding protein (GNBP-2). GDL is a simple, non-toxic and naturally occurring derivative of glucose. 
It was prepared to a final working solution of $300 \mathrm{mM}$ GDL and $100 \mathrm{mM}$ sodium acetate ( $\mathrm{NaOAc}$ ), pH 5.0 (Bulmer et al., 2009; Hamilton et al., 2011; Hamilton and Bulmer, 2012). An equivalent control solution containing only $100 \mathrm{mM}$ sodium acetate $(\mathrm{NaOAc})$, pH 5.0 was prepared. GDL or control solution were applied directly in the cellulose pad food source of the nest, with which colony nestmates had direct contact.

\section{Experimental Design}

Briefly, the $R$. flavipes mini-colonies were divided into control and GDL treatments after the 15-day colony establishment period had elapsed. Twenty-four hours prior to the introduction of the focal termites into mini-colonies, the paper food source inside every petri dish nest was moistened with $900 \mu \mathrm{l}$ of the GDL or control solution. Focal termites were comprised of either control (treated with $0.05 \%$ Tween 80 ) or infected (treated with $1 \times 10^{8}$ conidia/mL) individuals. Treatments are categorized from here on as follows: GDL+/ $\mathrm{Ma}-, \mathrm{GDL}-/$ $\mathrm{Ma}-, \mathrm{GDL}+/ \mathrm{Ma}+, \mathrm{GDL}-/ \mathrm{Ma}+$. For each of the treatments containing $M$. anisopliae there were 15 replicates (five per colony for three colonies) and nine replicates of the control treatments (three per colony for three colonies). We recorded behavioral responses of the experimental colonies to individuals treated with a lethal dose of the entomopathogenic fungus $M$. anisopliae or a Tween 80 control solution. Infected and control termites were added individually to the Petri dish nests and then resealed with parafilm. This took $\sim 9 \mathrm{~min}$, and the observation period began immediately after the last nest dish was sealed.

\section{Behavioral Recording}

We adopted the scan sampling method used in Davis et al. (2018). This form of instantaneous sampling allows for screening of multiple individuals (Altmann, 1974) and was used to observe the interactions between the focal termite and its nestmates. We recorded behavioral states at a single time-point during each scan of a focal termite in each experimental colony. Treatments were blinded and petri-dish locations were randomized prior to scanning. Scans typically took $<1$ min during which the location of the focal termite was identified and the observed behavioral state was immediately recorded. Where relevant, the number of groomers was quantified. A Samsung S7 smartphone was used as a digital voice recorder. Scans were performed every $5 \mathrm{~min}$ for a total of $3 \mathrm{~h}$. All observations were made at $27^{\circ} \mathrm{C}, 70 \%$ humidity under bright, constant overhead light. Experimental colonies were allowed to acclimatize to light for a period of $15 \mathrm{~h}$ prior to introduction of focal termites. Behaviors were classified into categories that are relevant to social immunity, and which are visually distinguishable and non-overlapping. As in Davis et al. (2018), we divided these behaviors into five different states:

Groomed by n: Focal termite is being groomed by n nestmates with no evidence of biting.

Cannibalism: Focal termite is being bitten by one or more nestmates and/or focal termite body is no longer intact.

Buried: Focal termite has had pieces of paper or feces placed on top of it. Although increasingly difficult to assess, the termite may still be alive.
Not visible: Focal termite is in a part of the nest where it cannot be observed.

Other: Focal termite is alive, intact, and unburied, but nestmates are not interacting with it. This reflects behavioral states unrelated to social immunity.

\section{Statistical Analysis}

All statistical analyses were performed using $\mathrm{R}$ version 3.6.0.

\section{Grooming}

Grooming amount(number of grooming states/total observed states) was analyzed by fitting a generalized linear mixed model using the glmer function in the $\mathrm{R}$ package lme4 v1.121 (Bates et al., 2015), employing a binomial error structure to account for proportion data (Crawley, 2014). The model was composed of an interaction between GDL and presence of $M$. anisopliae as a fixed effect, in addition to amount of cannibalism and colony. Petri dish nest ID was used as a random effect. The anova function was used to remove fixed effect parameters that did not lead to a significant alteration in deviance, as well as to perform likelihood ratio test comparisons. The final model was tested for overdispersion in the package blmeco v1.4 using the dispersion gmer function. We carried out post hoc pairwise comparisons using the glht function from the multcomp package v1.4-10 with Tukey correction (Korner-Nievergelt et al., 2015a,b).

Grooming intensity was analyzed with glmer to fit a generalized linear mixed model with a Poisson error structure, using total number of groomers in each experimental nest as the response variable. As before, the model was composed of an interaction between GDL and M. anisopliae presence as a fixed effect, in addition to amount of cannibalism and colony. Petri dish nest ID was used as a random effect. We logged the number of grooming states, and treated these as an offset to control for the fact that each observed grooming state increased the number of groomers by at least one. As before, we used anova to compare models. Again, post hoc pairwise comparisons were performed using glht with Tukey correction.

\section{Cannibalism}

To analyze whether GDL had an impact on time spent cannibalizing (number of cannibalism states/total observed states), we fitted a zero-inflated generalized linear mixed model using the glmmTMB function in the package glmmTMB v1.0.0 (Brooks et al., 2017) employing a binomial error structure to account for proportion data. We restricted our model to $\mathrm{GDL}+/ \mathrm{Ma}+$ and GDL-/Ma+, owing to insufficient data $(N=1$ observation of cannibalism) in experimental colonies exposed to control-treated focal individuals (GDL+/Ma- and GDL-/Ma-) and subsequent model convergence issues. The conditional component of the model contained GDL as a fixed effect, in addition to amount of grooming and colony. As before, petri dish nest ID was used as a random effect. The zero-inflation component of the model contained GDL as a fixed effect. Again, we used the anova function to inspect fixed effects, as well as to perform likelihood ratio test comparisons. Although GDL did not 


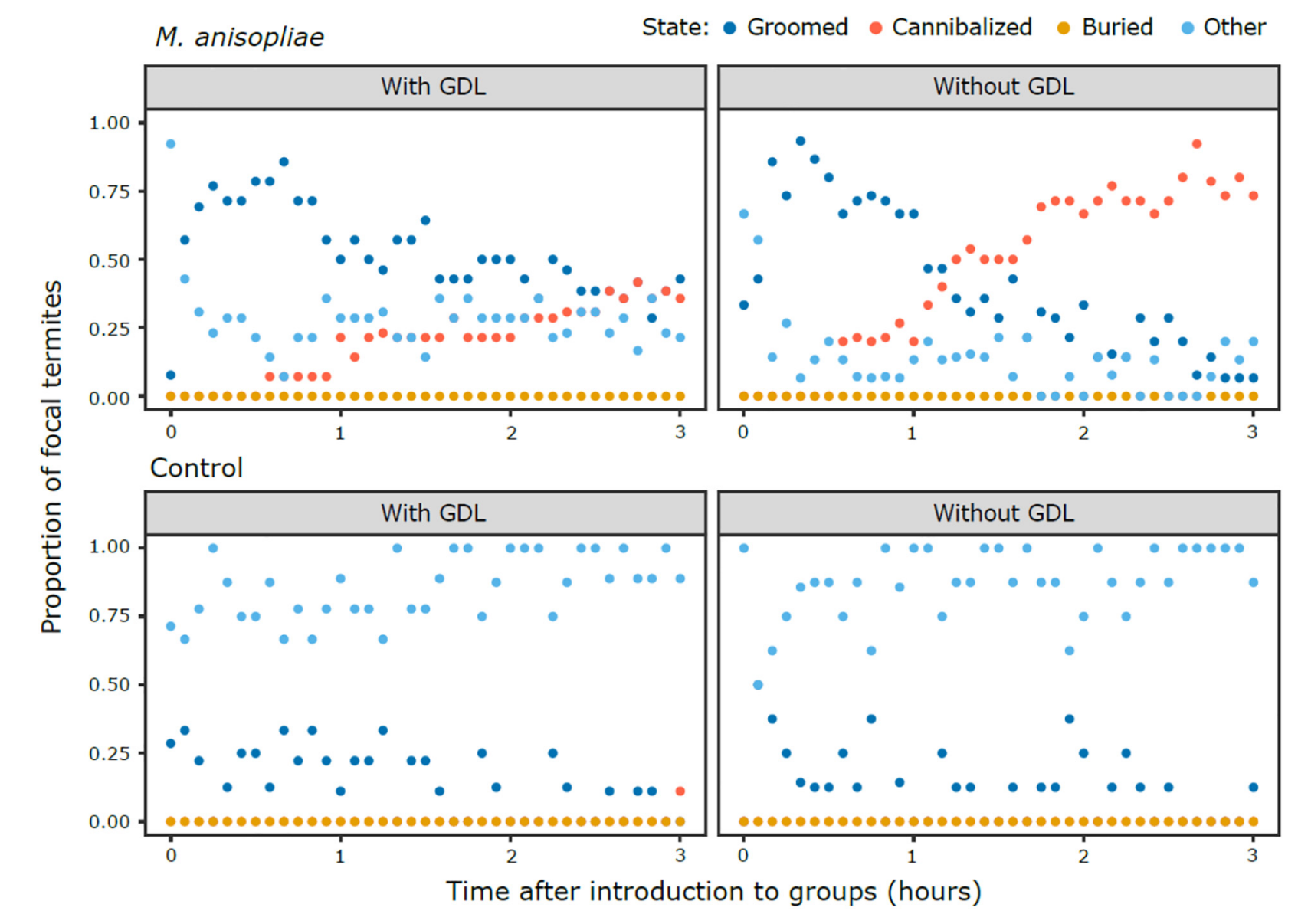

FIGURE 1 | Patterns of behavior over the $3 \mathrm{~h}$ scan period, showing M. anisopliae (top panels) and control treatments (bottom panels) in groups of nestmates exposed to GDL (left panels) or a control solution (right panels). The proportion of focal termites that are observed in a given state at each scan (conducted every $5 \mathrm{~min}$ ) are represented by a single point. The points overlap when more than one state was present at the same proportion across experimental colonies of a given treatment.

significantly improve the model when it was included as a factor in the conditional component of the model, its inclusion did slightly improve the distribution of residuals, and so was retained in the final model.

\section{RESULTS}

Following the exposure of focal termites to a control Tween 80 solution $(\mathrm{Ma}-)$ or $M$. anisopliae $(\mathrm{Ma}+)$ and isolating them for $12 \mathrm{~h}$, treated termites were introduced individually to experimental nests that had been exposed to GDL or a control NaOAc solution. Behavioral patterns (Figure 1) in the M. anisopliae absent groups (GDL-/ $\mathrm{Ma}-, \mathrm{GDL}+/ \mathrm{Ma}-$ ) were similar regardless of GDL treatment, in that they consisted mostly of behavioral states in the "other" category (states unrelated to social immunity), with low levels of grooming, one incident of cannibalism, and no observations of burial. Behavioral patterns in the GDL-/Ma+ groups after focal termites were introduced into the experimental colonies were characterized by a concentrated phase of grooming. Cannibalism began shortly thereafter, and almost completely replaced grooming before the end of the observation period. The GDL+/Ma+ groups were also typified by initially high levels of grooming, but the intensity of grooming slowly decreased over the course of the observation period. Although cannibalism was also observed this was largely at a lower level than in the GDL-/Ma+ groups and predominantly in the final hour of the scan. We did not observe burial in any of the treatments.

\section{GROOMING}

The amount of grooming was significantly higher in all $M$. anisopliae treatments compared with the controls (M. anisopliae treatments vs. corresponding controls: GDL-/Ma+ vs. GDL-/ Ma-, $z=7.399, P<0.001$; GDL-/Ma+ vs. GDL+/ Ma-, $z=6.861, P<0.001$; $\mathrm{GDL}+/ \mathrm{Ma}+$ vs. GDL+/ $\mathrm{Ma}-, \quad z=7.255, \quad P<0.001$; $\mathrm{GDL}+/ \mathrm{Ma}+$ vs. GDL-/ Ma-, $z=7.801, P<0.001$ ) (Figure 2 and Supplementary Table S1). The controls (GDL-/ $\mathrm{Ma}-$, $\mathrm{GDL}+/ \mathrm{Ma}-$ ) were not significantly different from each other 


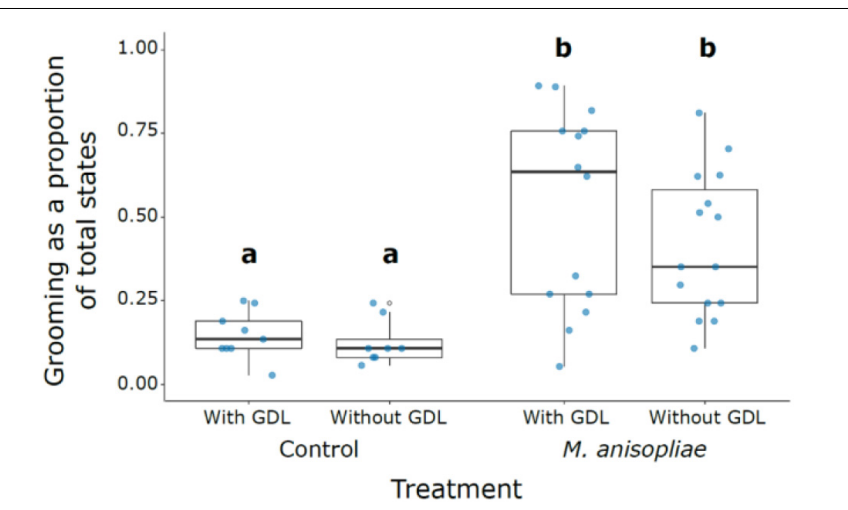

FIGURE 2 | Grooming as a proportion of total states across treatments. Different letters indicate treatments that were significantly different following post hoc comparison. First and third quartiles are indicated by the lower and upper hinges. Whiskers extend to the smallest/largest value when no smaller/greater than 1.5 times the inter-quartile range from the hinge.

and there was no significant effect of GDL treatment on proportion of grooming states. Low levels of grooming in $\mathrm{Ma}+$ treatments were significantly linked with a high proportion of cannibalism states $(z=-5.807, P<0.001)$ (Supplementary Figures S1, S2). The negative relationship between cannibalism and grooming in $\mathrm{Ma}+$ treatments may explain the trend towards an increased proportion of grooming in GDL-treated experimental colonies (Figure 2). We also found the amount of grooming to significantly depend on colony source, with colony $\mathrm{X}$ displaying higher grooming amounts than either of the other two colonies (Colony X vs. 10. $z=2.902, P<0.011$; Colony $X$ vs. $13+11, z=2.526, P=0.031$; Colony $13+11$ vs. $10, z=0.254$, $P=0.965)$ (Supplementary Figure S1). Intensity of grooming (number of groomers) was also significantly higher in all $M$. anisopliae treatments over the controls ( $M$. anisopliae treatments vs. corresponding controls: GDL-/Ma+ vs. GDL-/Ma-. $z=3.603 . P<0.002 ; \mathrm{GDL}-/ \mathrm{Ma}+$ vs. GDL+/Ma-. $z=3.213, P<0.007$; GDL+/Ma+ vs. GDL+/ Ma-, $z=3.676$, $P<0.002 ; \mathrm{GDL}+/ \mathrm{Ma}+$ vs. GDL-/ Ma-, $z=4.015, P<0.001)$ (Supplementary Figure S3 and Supplementary Table S2). The controls (GDL-/ $\mathrm{Ma}-, \mathrm{GDL}+/ \mathrm{Ma}-$ ) were not significantly different from each other and there was no significant effect of GDL treatment on number of groomers. Grooming intensity and number of groomers increased sharply in both M. anisopliae treatments following the introduction of focal termites, particularly in the GDL-/Ma+ treatment (Figure 3). $\mathrm{Ma}+$ groups lacking GDL also exhibited a sharper decline in both intensity and number of groomers over the course of the observation period, as grooming states were gradually replaced with cannibalism (Figure 1). In contrast to amount of grooming, high numbers of groomers in $\mathrm{Ma}+$ treatments were significantly associated with a high proportion of cannibalism states $(z=2.651, P=0.008)$. Higher numbers of groomers were also observed in colony $\mathrm{X}$ compared with the remaining two colonies (Colony X vs. $10, z=2.547, P=0$. 0291; Colony X vs. $13+11, z=3.222, P=0.004$; Colony $13+11$ vs. $10, \mathrm{z}=-0.788$, $P=0.71024)$ (Supplementary Figures S4, S5).

\section{Cannibalism}

The probability of being harmed during the observation period following exposure to $M$. anisopliae was significantly reduced by the inhibition of GNBP-2 (zero inflation term, GDL- vs. GDL $+, z=2.218, P=0.027$ ) (Figure 4 and Supplementary Table S3). Furthermore, amount of cannibalism was negatively associated with amount of grooming $(z=-9.053, P<0.001)$. Cannibalism also varied significantly by colony, with colony $13+11$ displaying lower amounts of cannibalism than either colonies 10 and $\mathrm{X}$ (Colony X vs. 10, $z=-0.164, P=0.985$; Colony $\mathrm{X}$ vs. $13+11, z=2.744, P=0.017$; Colony $13+11$ vs. 10 , $z=-3.041, P=0.007$ ) (Supplementary Figures S6, S7).

\section{DISCUSSION}

GDL treatment resulted in suppression of pathogen-induced cannibalistic behavior. But contrary to our expectations, the amount and intensity of grooming was not influenced by the application of GDL. This indicates that GNBP-2 glucanase activity can stimulate the transition from intense grooming to cannibalism but appears not to play a major role in the initial stimulation of grooming or in acting to recruit more groomers. Yanagawa et al. (2011) found that the filtrate from a suspension of $M$. anisopliae conidia was enough to induce grooming in Coptotermes formosanus, suggesting that grooming can be induced by the presence of fungal pathogen-associated molecular signatures (PAMPs). Interestingly, the same study did not detect any evidence of enhanced cannibalism, indicating that these are behaviors induced by signals released after infection. Davis et al. (2018) confirmed this suspicion by showing that defensive cannibalism only takes place once an infection has yielded an explicit sickness response in the termite host. In the same study, grooming was found to increase after conidia had begun to germinate, becoming even more elevated once hosts began to display signs of sickness. Similarly, in a study on ants, Pull et al. (2018) showed that pupae-derived chemical cues are used by workers to target infected pupae for destruction with poison spraying. These findings suggest that fungal PAMPs in combination with host-derived stimuli drive both grooming and destructive disinfection behaviors in social insects, as well as regulating the transition between these states. The data from the current study suggest that while GNBP-2 is unlikely to be the main mechanism by which termites detect fungal PAMPs, its activity can nonetheless influence collective behavior once the host has become sick, potentially via the release of fungal PAMPs from damaged host cuticle. It is possible that GNBP-2 inhibition does not strongly discourage grooming because termites could employ a variety of host and/or pathogen-derived signals, involving behavioral, chemical or even oscillatory cues (e.g., body vibrations) to initiate collective defense tasks (Rosengaus et al., 1999; Wilson-Rich et al., 2007; Zhukovskaya et al., 2013; Davis et al., 2018; Bulmer et al., 2019). In this scenario, although GNBP2 activity may itself accelerate the transition from a caring to a killing response, it represents just one component of a complex repertoire of social immune mechanisms that termites could use to regulate infectious threats exposed to the colony. Given 


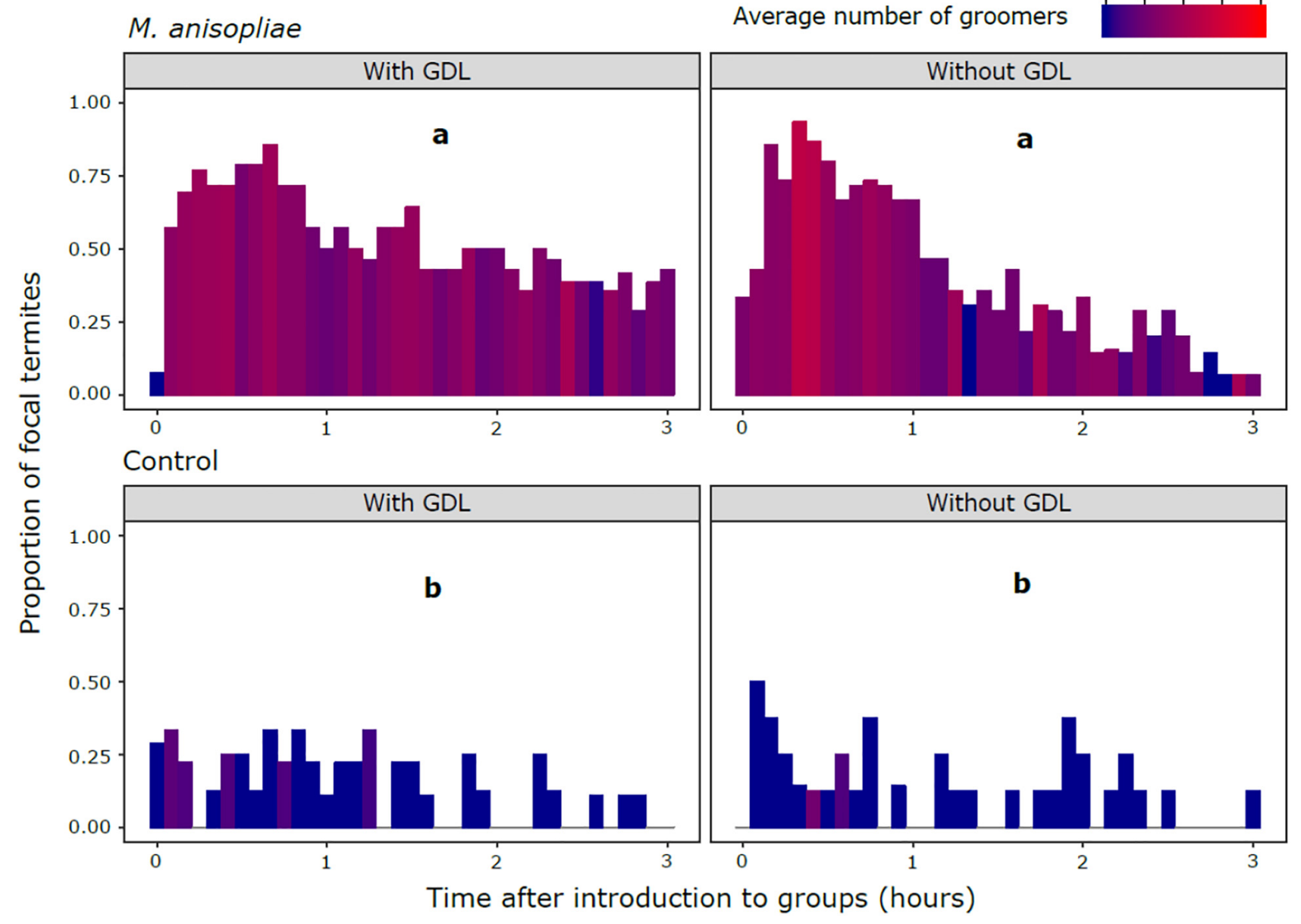

FIGURE 3 | Proportion of focal termites observed being groomed by nestmates in each scan. M. anisopliae (top panels) and control treatments (bottom panels) in groups of nestmates exposed to GDL (left panels) or a control solution (right panels). Different letters indicate treatments in which overall number of groomers were significantly different following post hoc comparisons, after accounting for number of grooming states. Color of the fill represents the average number of groomers at each time point.

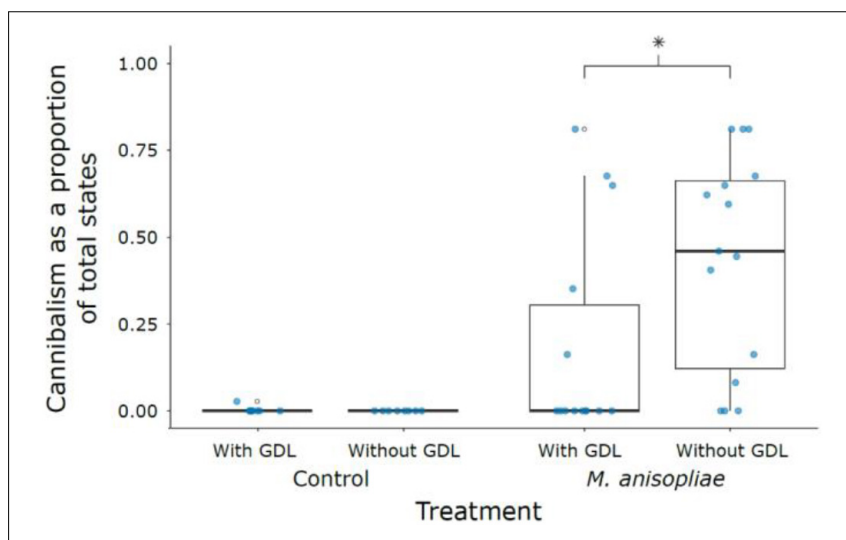

FIGURE 4 | Cannibalism as a proportion of total states across treatments. Absence of cannibalism in M. anisopliae groups depends significantly on GDL treatment (indicated by an asterisk). First and third quartiles are indicated by the lower and upper hinges. Whiskers extend to the smallest/largest value when no smaller/greater than 1.5 times the inter-quartile range from the hinge. the additional function of GNBP-2 as an internal disinfectant and putative regulator of gut symbiosis, the observed behavioral shift could represent an individual feedback response linked to disrupted digestion, rather than as a regulator of social immunity. However, it is also conceivable that GNBP-2 could fulfill both functions simultaneously.

While many studies underline the importance of collective defenses in preventing pathogen infection in termite colonies (Rosengaus et al., 1998; Traniello et al., 2002; Yanagawa and Shimizu, 2007; Zhukovskaya et al., 2013) this is to our knowledge the first to show a link between an immune molecule and collective behavioral defense. Social immune behaviors are described in several insect societies and their role in preventing emerging infectious diseases is now an established field of research (Cremer et al., 2007, 2018; Cotter and Kilner, 2010; Meunier, 2015; Kennedy et al., 2017). In addition to representing an effective model for social immunity research, our study highlights the importance of termites as a key comparative lineage to the social Hymenoptera, particularly ants, which have 
been a favored model for investigations into social immunity (Hughes et al., 2002; Baer et al., 2005; Ugelvig et al., 2010; Reber et al., 2011; Walker and Hughes, 2011; Pull et al., 2018). It would be particularly interesting to understand whether convergent social immune mechanisms have evolved in independent eusocial and superorganismal hymenopteran lineages. An expectation might be that externally secreted antimicrobial compounds or immune molecules can also influence collective hygienic behaviors in such groups, in addition to acting as straightforward external disinfectants.

\section{CONCLUSION}

In recent years, researchers have been trying to understand the relationships between the different layers of the social immune system: from internal physiological defenses, to the secretion of antimicrobial compounds, and culminating in the careful coordination of collective defensive behaviors. These studies are focused mainly on their evolution (Harpur and Zayed, 2013; Otti et al., 2014; Meunier, 2015; Cremer et al., 2018; Van Meyel et al., 2018) or in understanding resource allocation among the different levels of immunity to discover possible trade-offs (Armitage and Boomsma, 2010; Cotter et al., 2013; Rosengaus et al., 2013; Harpur et al., 2014; Gao and Thompson, 2015). Our aim in this study was to experimentally test the functional relationship between these different immune layers, with the specific goal of exploring whether the "care or kill" collective defense response of a termite could be influenced by the inhibition of the fungicidal immune enzyme, GNBP-2. Although GNBP-2 represents just one piece of a larger puzzle, our findings indicate that different components of the social immune system may interact with one another. Our study describes how the orchestration of group-level hygienic behaviors could rely at least in part on relatively simple cues mediated by externally secreted molecules from the individual immune system.

\section{REFERENCES}

Altmann, J. (1974). Observational study of behavior: sampling methods. Behaviour 49, 227-267.

Armitage, S. A. O., and Boomsma, J. J. (2010). The effects of age and social interactions on innate immunity in a leaf-cutting ant. J. Insect. Physiol. 56, 780-787. doi: 10.1016/j.jinsphys.2010.01.009

Baer, B., Krug, A., Boomsma, J. J., and Hughes, W. O. H. (2005). Examination of the immune responses of males and workers of the leaf-cutting ant Acromyrmex echinatior and the effect of infection. Insect. Soc. 52, 298-303. doi: 10.1007/ s00040-005-0809-x

Bates, D., Mächler, M., Bolker, B., and Walke, S. (2015). Fitting linear mixed-effects models using lme4. J. Stat. Softw. 67. doi: 10.18637/jss.v067.i01

Becker, G. (1969). "Rearing of termites and testing methods used in the laboratory," in Biology of Termites, eds K. Krishna and F. M. Weesner (New York, NY: Academic Press), 351-385. doi: 10.1016/B978-0-12-395529-6.50015-5

Brooks, M. E., Kasper, K., Koen, B. J., Arni, M. V., Casper, B. W., Anders, N., et al. (2017). GlmmTMB balances speed and flexibility among packages for zeroinflated generalized linear mixed modeling. $R$ J. 9, 378-400. doi: 10.32614/RJ2017-2066

Bulmer, M. S., Franco, B. A., and Fields, E. G. (2019). Subterranean termite social alarm and hygienic responses to fungal pathogens. Insects 10:240. doi: 10.3390/ insects 10080240

\section{DATA AVAILABILITY STATEMENT}

All datasets generated for this study are included in the article/Supplementary Material.

\section{AUTHOR CONTRIBUTIONS}

DM and SM conceived the study. ME-M and HD carried out the experiments. ME-M, HD, and DM analyzed the data. ME$\mathrm{M}$ and DM wrote the manuscript. All authors were involved in editing the manuscript.

\section{FUNDING}

Open Access Funding provided by the Freie Universität Berlin.

\section{ACKNOWLEDGMENTS}

We acknowledge J. Rolff for discussion and support during the experiment. This study was supported by BASF-Wolman GmbH; a DAAD Research Grant for Doctoral Candidates and Young Academics to SM; and a DAAD Doctoral Scholarship to ME-M.

\section{SUPPLEMENTARY MATERIAL}

The Supplementary Material for this article can be found online at: https://www.frontiersin.org/articles/10.3389/fevo.2020.00075/ full\#supplementary-material

DATA SHEET S1 | Raw data.

DATA SHEET $\mathrm{S} 2 \mid \mathrm{R}$ code.

PRESENTATION S1 | Supplementary Information (Supplementary

Figures S1-S7 and Supplementary Tables S1-S3).

Bulmer, M. S., Ido, B., Rahul, R., Rosengaus, R. B., and Ram, S. (2009). Targeting an antimicrobial effector function in insect immunity as a pest control strategy. PNAS 106, 12652-12657. doi: 10.1073/pnas.0904063106

Chouvenc, T., and Su, N. Y. (2010). Apparent synergy among defense mechanisms in subterranean termites (Rhinotermitidae) against epizootic events: limits and potential for biological control. J. Econ. Entomol. 103, 1327-1337. doi: 10.1603/ ec09407

Chouvenc, T., and Su, N. Y. (2012). When subterranean termites challenge the rules of fungal epizootics. PLoS One 7:e34484. doi: 10.1371/journal.pone.0034484

Chouvenc, T., Su, N. Y., and Alain, R. (2009). Inhibition of Metarhizium anisopliae in the alimentary tract of the Eastern subterranean termite Reticulitermes flavipes. J. Invertebr. Pathol. 101, 130-136. doi: 10.1016/j.jip.2009.04.005

Chouvenc, T., Su, N. Y., and Elliott, M. (2008). Interaction between the subterranean termite Reticulitermes flavipes (Isoptera: Rhinotermitidae) and the entomopathogenic fungus Metarhizium anisopliae in foraging arenas. J. Econ. Entomol. 101, 885-893. doi: 10.1093/jee/101.3.885

Cotter, S. C., and Kilner, R. M. (2010). Personal immunity versus social immunity. Behav. Ecol. 21, 663-668. doi: 10.1093/beheco/arq070

Cotter, S. C., Littlefair, J. E., Grantham, P. J., and Kilner, R. M. (2013). A direct physiological trade-off between personal and social immunity. J. Anim. Ecol. 82, 846-853. doi: 10.1111/1365-2656.12047

Crawley, M. J. (2014). Statistics: An Introduction Using R, 2nd Edn, Chichester: John Wiley \& Sons, Ltd. 
Cremer, S., Armitage, S. A. O., and Schmid-Hempel, P. (2007). Social immunity. Curr Biol. 17, R693-R702. doi: 10.1016/j.cub.2007.06.008

Cremer, S., Pull, C. D., and Fürst, M. A. (2018). Social immunity: emergence and evolution of colony-level disease protection. Annu. Rev. Entomol. 63, 105-123. doi: 10.1146/annurev-ento-020117-043110

Davis, H. E., Meconcelli, S., Radek, R., and McMahon, D. P. (2018). Termites shape their collective behavioural response based on stage of infection. Sci. Rep. 8:14433. doi: 10.1038/s41598-018-32721-32727

Dong, C., Jiamin, Z., Wuguo, C., Hai, H., and Yuanyang, H. (2007). Characterization of a newly discovered china variety of Metarhizium anisopliae (M. anisopliae Var. Dcjhyium) for virulence to termites, isoenzyme, and phylogenic analysis. Microbiol. Res. 162, 53-61. doi: 10.1016/j.micres.2006. 07.001

Evans, T. A. (2000). Fast marking of termites (Isoptera: Rhinotermitidae). Sociobiology 36, 517-523.

Gao, Q., and Thompson, G. J. (2015). Social context affects immune gene expression in a subterranean termite. Insect. Soc. 62, 167-170. doi: 10.1007/ s00040-015-0389-383

Hamilton, C., and Bulmer, M. S. (2012). Molecular antifungal defenses in subterranean termites: RNA interference reveals in vivo roles of termicins and GNBPs against a naturally encountered pathogen. Dev. Comp. Immunol. 36, 372-377. doi: 10.1016/j.dci.2011.07.008

Hamilton, C., Lay, F., and Bulmer, M. S. (2011). Subterranean termite prophylactic secretions and external antifungal defenses. J. Insect. Physiol. 57, 1259-1266. doi: 10.1016/j.jinsphys.2011.05.016

Harpur, B. A., Chernyshova, A., Arash, S., Nadejda, T., Mohammad, M., Zhixing, X., et al. (2014). No genetic tradeoffs between hygienic behaviour and individual innate immunity in the honey bee, Apis mellifera. PLoS One 9:e104214. doi: 10.1371/journal.pone.0104214

Harpur, B. A., and Zayed, A. (2013). Accelerated evolution of innate immunity proteins in social insects: adaptive evolution or relaxed constraint? Mol. Biol. Evol. 30, 1665-1674. doi: 10.1093/molbev/mst061

He, S., Johnston, P. R., Kuropka, B., Lokatis, S., Weise, C., Plarre, R., et al. (2018). Termite soldiers contribute to social immunity by synthesizing potent oral secretions. Insect. Mol. Biol. 27, 564-576. doi: 10.1111/imb.12499

Hughes, W. O. H., Jørgen, E., and Boomsma, J. J. (2002). Trade-offs in group living: transmission and disease resistance in leaf-cutting ants. Proc. R. Soc. Lond. B. 269, 1811-1819. doi: 10.1098/rspb.2002.2113

Kennedy, P., Baron, G., Qiu, B., Freitak, D., Helanterä, H., Hunt, E. R., et al. (2017). Deconstructing superorganisms and societies to address big questions in biology. Trends Ecol. Evol. 32, 861-872. doi: 10.1016/j.tree.2017. 08.004

Konrad, M., Meghan, L., Vyleta, F. J. T., Miriam, S., Tragust, S., Klatt, M., et al. (2012). Social transfer of pathogenic fungus promotes active immunisation in ant Colonies. PLoS Biol. 10:e1001300. doi: 10.1371/journal.pbio. 1001300

Konrad, M., Pull, C. D., Sina, M., Katharina, S., Naderlinger, E., Grasse, A. V., et al. (2018). Ants avoid superinfections by performing risk-adjusted sanitary care. PNAS 115, 2782-2787. doi: 10.1073/pnas.1713501115

Korner-Nievergelt, F., Roth, T., von Felten, S., Guélat, J., Almasi, B., and KornerNievergelt, P. (2015a). Bayesian Data Analysis in Ecology Using Linear Models with R, BUGS, and Stan. Cambridge, MA: Academic Press, doi: 10.1016/C20130-23227-X

Korner-Nievergelt, F., Roth, T., von Felten, S., Guélat, J., Almasi, B., and KornerNievergelt, P. (2015b). Bayesian Data Analysis In Ecology Using Linear Models With R, BUGS and Stan. London: Elsevier.

Lainé, L., and Wright, D. (2003). The life cycle of Reticulitermes spp (Isoptera: Rhinotermitidae): what do we know? Biol. Entomol. Res. 93, 267-278. doi: 10.1079/BER2003238

Lamberty, M., Zachary, D., Lanot, R., Bordereau, C., Robert, A., Hoffmann, J. A., et al. (2001). Insect immunity constitutive expression of a cysteine-rich antifungal and a linear antibacterial peptide in a termite insect. J. Biol. Chem. 276, 4085-4092. doi: 10.1074/jbc.m002998200

Liu, L., Xing-Ying, Z., Qing-Bo, T., Chao-Liang, L., and Qiu-Ying, H. (2019). The mechanisms of social immunity against fungal infections in eusocial insects. Toxins 11:244. doi: 10.3390/toxins11050244

López-Riquelme, G. O., and Fanjul-Moles, M. L. (2013). The funeral ways of social insects. Soc. Strateg. Corpse Disposal. Entomol. 9, 71-129.
Meunier, J. (2015). Social immunity and the evolution of group living in insects. Philos. Trans. R. Soc. B. 370:20140102. doi: 10.1098/rstb.2014.0102

Mora, M. A., Conteiro, A. M., and Fraga, M. E. (2017). Classification and infection mechanism of entomopathogenic fungi. Arq. Inst. Biol. 84, 0552015. doi: 10. 1590/1808-1657000552015

Otti, O., Tragust, S., and Feldhaar, H. (2014). Unifying external and internal immune defences. Trends Ecol. Evol. 29, 625-634. doi: 10.1016/j.tree.2014. 09.002

Pull, C. D., Ugelvig, L. V., Wiesenhofer, F., Grasse, A. V., Tragust, S., Schmitt, T., et al. (2018). Destructive disinfection of infected brood prevents systemic disease spread in ant colonies. eLife 7:e32073. doi: 10.7554/eLife. 32073

Reber, A., Purcell, J., Buechel, S. D., Buri, P., and Chapuisat, M. (2011). The expression and impact of antifungal grooming in ants. J. Evol. Biol. 24, 954-964. doi: $10.1111 / j .1420-9101.2011 .02230 . x$

Richard, D. A. (1974). The evolution of social behavior. Annu. Rev. Ecol. Syst. 5, 325-383. doi: 10.1146/annurev.es.05.110174.001545

Rosengaus, R. B., Jordan, C., Lefebvre, M. L., and Traniello, J. F. A. (1999). Pathogen alarm behavior in a termite: a new form of communication in social insects. Naturwissenschaften 86, 544-548. doi: 10.1007/s00114005 0672

Rosengaus, R. B., Kelley, F. S., Alla, Y., Bulmer, M. S., William, S. D., Ryan, W. B., et al. (2014). Symbiont-Derived $\beta-1,3-$ Glucanases in a social insect: mutualism beyond Nutrition. Front. Microbiol. 5:607. doi: 10.3389/fmicb.2014. 00607

Rosengaus, R. B., Malak, T., and MacKintosh, C. (2013). Immune-priming in ant larvae: social immunity does not undermine individual immunity. Biol. Lett. 9:20130563. doi: 10.1098/rsbl.2013.0563

Rosengaus, R. B., Maxmen, A. B., Coates, L. E., and Traniello, J. F. A. (1998). Disease resistance: a benefit of sociality in the dampwood termite Zootermopsis Angusticollis (Isoptera: Termopsidae). Behav. Ecol. Sociobiol. 44, 125-134. doi: $10.1007 / \mathrm{s} 002650050523$

Rosengaus, R. B., Traniello, J. F. A., and Bulmer, M. S. (2011). "Ecology, behavior and evolution of disease resistance in termites," in Biology of Termites: A Modern Synthesis, eds D. E. Bignell, Y. Roisin, and L. Nathan (Dordrecht: Springer), 165-191. doi: 10.1007/978-90-481-3977-4-7

Schmid-Hempel, P. (1995). Parasites and social insects. Apidologie 26, 255-271. doi: 10.1051/apido:19950307

Schmid-Hempel, P. (2017). Parasites and their social hosts. Trends Parasitol. 33, 453-466. doi: 10.1016/j.pt.2017.01.003

Tracy, Z. D. G. (2003). Sampling subterranean termite species diversity and activity in tropical savannas: an assessment of different bait choices. Ecol. Entomol. 28, 397-404. doi: 10.1046/j.1365-2311.2003.00525.x

Traniello, J. F. A., Rosengaus, R. B., and Keely, S. (2002). The development of immunity in a social insect: evidence for the group facilitation of disease resistance. PNAS 99, 6838-6842. doi: 10.1073/pnas.102176599

Ugelvig, L. V., Kronauer, D. J. C., Schrempf, A., Heinze, J., and Cremer, S. (2010). Rapid anti-pathogen response in ant societies relies on high genetic diversity. Proc. R. Soc. B 277, 2821-2828. doi: 10.1098/rspb.2010.0644

Van Meyel, S., Körner, M., and Meunier, J. (2018). Social immunity: why we should study its nature, evolution and functions across all social systems. Curr. Opin. Insect. Sci. 28, 1-7. doi: 10.1016/j.cois.2018.03.004

Vargo, E., and Husseneder, C. (2009). Biology of subterranean termites: insights from molecular studies of Reticulitermes and Coptotermes. Annu. Rev. Entomol. 54, 379-403. doi: 10.1146/annurev.ento.54.110807.090443

Vega, F. E., Meyling, N. V., Luangsa-ard, J. J., and Blackwell, M. (2012). "Chapter 6 - Fungal Entomopathogens," in Insect Pathology, eds F. E. Vega and H. K. Kaya (San Diego: Academic Press), 171-220. doi: 10.1016/B978-0-12-3849847.00006-3

Walker, T. N., and Hughes, W. O. H. (2011). Arboreality and the evolution of disease resistance in ants. Ecol. Entomol. 36, 588-595. doi: 10.1111/j.1365-2311. 2011.01312.x

Wilson-Rich, N., Spivak, M., Fefferman, N. H., and Starks, P. T. (2009). Genetic, individual, and group facilitation of disease resistance in insect societies. Annu. Rev. Entomol. 54, 405-423. doi: 10.1146/annurev.ento.53.103106. 093301

Wilson-Rich, N., Stuart, R., and Rosengaus, R. B. (2007). Susceptibility and behavioral responses of the dampwood termite Zootermopsis angusticollis to the 
entomopathogenic nematode Steinernema carpocapsae. J. Invertebr. Pathol. 95, 17-25. doi: 10.1016/j.jip.2006.11.004

Yanagawa, A., Fujiwara-Tsuji, N., Akino, T., Yoshimura, T., Yanagawa, T., and Shimizu, S. (2011). Musty odor of entomopathogens enhances diseaseprevention behaviors in the termite Coptotermes formosanus. J. Invertebr. Pathol. 108, 1-6. doi: 10.1016/j.jip.2011.06.001

Yanagawa, A., Fumio, Y., and Shimizu, S. (2008). Defense mechanism of the termite, Coptotermes formosanus shiraki, to entomopathogenic fungi. J. Invertebr. Pathol. 97, 165-170. doi: 10.1016/j.jip.2007.09.005

Yanagawa, A., and Shimizu, S. (2007). Resistance of the termite, Coptotermes formosanus Shiraki to Metarhizium anisopliae due to grooming. Biocontrol 52, 75-85. doi: 10.1007/s10526-006-9020-x

Yuki, M., Shigeharu, M., Tetsushi, I., and Toshiaki, K. (2008). Transcriptome analysis of the digestive organs of Hodotermopsis sjostedti, a lower termite that hosts mutualistic microorganisms in its hindgut. Zool. Sci. 25, 401-406. doi: 10.2108 /zsj.25.401

Zasloff, M. (2002). Antimicrobial peptides of multicellular organisms. Nature 415:389. doi: $10.1038 / 415389$ a
Zhukovskaya, M., Yanagawa, A., and Forschler, B. T. (2013). Grooming behavior as a mechanism of insect disease defense. Insects 4, 609-630. doi: 10.3390/ insects4040609

Zoberi, M. H. (1995). Metarhizium anisopliae, a fungal pathogen of Reticulitermes flavipes (Isoptera: Rhinotermitidae). Mycologia 87, 354-359. doi: 10.1080/ 00275514.1995 .12026539

Conflict of Interest: The authors declare that the research was conducted in the absence of any commercial or financial relationships that could be construed as a potential conflict of interest.

Copyright (C) 2020 Esparza-Mora, Davis, Meconcelli, Plarre and McMahon. This is an open-access article distributed under the terms of the Creative Commons Attribution License (CC BY). The use, distribution or reproduction in other forums is permitted, provided the original author(s) and the copyright owner(s) are credited and that the original publication in this journal is cited, in accordance with accepted academic practice. No use, distribution or reproduction is permitted which does not comply with these terms. 